\title{
Archival Files Consulted
}

\section{Tashkent}

TsGARUz, f. I-1 [Kantseliariia Turkestanskogo General-Gubernatora], op. 2, d. 1023: O naznachenii pensii byvshemu Katta-Kurganskomu narodnomu sud'i Mulla-Akhmetu Mulla Adinaevu, 19.12.1908.

TsGARUz, f. I-1, op. 11, d. 326: Ob uchrezhdenii komissii dlia obsuzhdeniia voprosa ob ustroistve dukhovnogo upravleniia musul'man i organizatsii vakufnykh uchrezhdenii turkestanskogo kraia, 1884-5.

TsGARUz, f. I-1, op. 12, d. 44: Po khodataistvu vakufnogo ustanovleniia Medrese Padshcha Iskander Kukhna v gor. Star. Margelane o prieme v kaznu prinadlezhashchego im uchastka naselennoi vakufnoi zemli ploshchad'iu $v$ 1541 desiatin $i$ voobshche o prave vakufnykh uchrezdenii otchuzhdat' prinadlezhashchie im nedvizhnie imushchestva i o prave vladel'tsev voobshche na otkaz ot prinadlezhashchikh im zemel' posle proizvodstva pozemel'no podatnykh rabot, 1900-1.

TsGARUz, f. I-1, op. 12, d. 430: Po prosheniiu Gafurbaia Magdalinova ob uprazdnenii uchrezhdennogo ego ottsom vakufa Magdali-Supivg. Osh, 1903-4.

TsGARUz, f. I-1, op. 12, d. 900: Po prosheniiu doverennogo tuzemtsa Mulla MirUmara Khodzhi Mir-Salimova, Mikhaila Ivanovicha Raikova khodataistvuiushchego o nepriznanii vakufom dvukh uchastkov zemli, 1906.

TsGARUz, f. I-1, op. 14, d. 28: O vakufnykh i mil'kovykh zemliakh, nakhodiashchikhsia $v$ Zeravshanskom okruge o poriadke raskhodovaniia postupaiushchikh s ètikh zemel' dokhodov, 1869-70.

TsGARUz, f. I-1, op. 16, d. 66: Zhurnaly zasedanii Tashkentskoi organisatsionnoi komissii $1868 \mathrm{~g}$.

TsGARUz, f. I-1, op. 22, d. 3: S dokladom komissii po peresmotru i izmeneniiu proekta "Polozheniia ob upravlenii v semirechenskoi obl. i syr-dar'inskoi obl.," 1870.

TsGARUz, f. I-1, op. 25, d. 107: Protokoly zasedaniia komissii uchrezhdennoi prikazom po Turkestanskomu kraiu ot 5 iiunia 1904 za no. 147 po rassmotreniiu voprosov, kasaiushchikhsia postonovki vakufnogo dela v Turkestanskom krae, 1904.

TsGARUz, f. I-1, op. 27, d. 68: Ob ustroistve sudebnoi chastiv Turkestanskom krae i ob'iasnitel'naia zapiska o gornom upravlenii v Turkestanskom krae, 1881.

TsGARUz, f. I-2 [Diplomaticheskii chinovnik pri Turkestanskom General Gubernatore] op. 1 d. 314: Nil S. Lykoshin, Zapiska Amu-Dar'inskogo otdela Polkovnika Lykoshina o sovremennom sostoianii Khivinskogo khanstva, 1912. 
TsGARUz, f. I-17, [Syr-Dar'inskoe oblastnoe pravlenie] op. 1, d. 2059: O naznachenii Kantselarskogo Sluzhitelia Perovskogo Uezdnogo Upravleniia neimeiushchogo china Seita Akbergen o nepravil'nykh deistviiakh nar. sud. eva [sic] slovesnim perevodchikom Perovskogo Uezdnogo Upravleniia, 1911.

TsGARUz, f. I-17, op. 1, d. 2850: Ob opredelenii na Gosudarstvennuiu sluzhbu Mirza Radzhaba Abdudzhabarova, 1909.

TsGARUz, f. I-17, op. 1, d. 4784: O khodataistvakh advokatov po delam, razbyraemym po shariatu, 1886 .

TsGARUz, f. I-17, op. 1, d. 4784: O nepravil'nykh deistviiakh nar. sud. Mukhiddinkhodzha, 1891.

TsGARUz, f. I-17, op. 1, d. 4887: Po obvineniiu Kaziia Sibzarskoi chasti goroda Tashkenta Mukhitdina khodzhi Tashkenstkim sartom Sadykdzhanom Usta Akhundzhanovym v prisvoenii prav mutavaliia nad vakufnym imushchestvom Turabaevoi, 1892.

TsGARUz, f. I-17, op. 1, d. 5387: Po obvineniiu kaziia Sibzarsoi chasti Mukhiddinkhodzhi, 1894.

TsGARUz, f. I-17, op. 1, d. 5775: Po obvineniiu kaziia Sibzarsoi chasti Mukhiddinkhodzhi, 1896.

TsGARUz, f. I-17, op. 1, d. 6226: Po obvineniiu kazia Sibzarskoi chasti Mukhiddinkhodzhi i muftiia Sultan Iunusmukhammedova po 362 st., 1897.

TsGARUz, f. I-17, op. 1, d. 6366: Po obvineniiu kaziia Sibzarskoi chasti Mukhiddinkhodzhi o nepravil'nykh deistviiakh, 1898.

TsGARUz, f. I-17, op. 1, d. 6367: Po obvineniiu kaziia Sibzarskoi chasti Mukhiddinkhodzhi o nezakonnykh deistviiakh, 1898.

TsGARUz, f. I-17, op. 1, d. 6468: Po obvineniiu Mukhitdina Khodzhi po 354 i 352 st., 1898.

TsGARUz, f. I-17, op. 1, d. 6469: Po obvineniiu byv. nar. sud. Sibzarskoi chasti Mukhiddinkhodzhi po sluzhbe, n.d.

TsGARUz, f. I-17, op. 1, d. 30291/23: Po voprosu o tom imeiut li silu iuridischeskogo mezhevaniia raboty byvsh. pozemel'no-podatnoi komissii i mogut li byt' premeniaemy i knim vse ukazaniia mezhevykh zakonov, n.d.

TsGARUz, f. I-17, op. 1, d. 31916: Spiski vakufov nakhodiashchikhsia v cherte gorodov, 1888.

TsGARUz, f. I-17, op. 1, d. 32587: Po proizvedennomu komissarom 5-go pozemel'nopodatnago uchastka izsledovaniiu dokumentov i imushchestva, otnosiashchikhsia kvakufu medresse "Issa-Khodzha Kazy-Keliana," 1892.

TsGARUz, f. I-17, op. 1, d. 32597: Vakuf mecheti kvartala Kar-Iagdy, v g. Tashkente $s$ vakufnym dokumentom mecheti kvartala Kar Iagdy, predstavlennym mutavaliem Khamra Khodzha Tiura Khodzhinovym, 1887-8. 
TsGARUz, f. I-17, op. 1, d. 32607: Vakuf dvukh mechetei kvartala Makhsiduz v g. Tashkente. $S$ vakufnymi dokumentami dvukh mechetei kvartala Makhsiduz, predostavlennym mutavaliem Zakirdzhanom Akhundzhanovym, 1887-88.

TsGARUz, f. I-17, op. 1, d. 32663: Vakufnye dokumenty medresse nakhodiashchikhsiav gor. Tashkente, n.d.

TsGARUz, f. I-17, op. 1, d. 35430: Posluzhnoi spisok pis'mennogo perevodchika pri Upravlenii Nachal'nika goroda Tashkenta, china Shakirdzhana Ishaeva, 1901.

TsGARUz, f. I-18 [Samarkandskoe Oblastnoe Pravlenie], op. 1, d.139: N. Mordvinov, Zapiska k proektu o sudebnoi reforme $v$ Turkestanskom krae, 1891.

TsGARUz, f. I-19 [Ferganskoe Oblastnoe Pravlenie], op. 1, d. 3498: untitled.

TsGARUz, f. I-19, op. 1, d. 33346: Po vozbuzhdennomu upravliaiushchei Turkestanskoiu Kazennoiu Palatoiu voprosu kakoe naznachenie dolzhny poluchit' te vakufnye imushchestva, za kotorymi vakufnoe pravo ne budet priznano, 1893 .

TsGARUz, I-21 [Upravlenie Nachal'nika Dzhizakskogo Uezda, Samarkandskoi Oblasti], op. 1, d. 56: O spore iz-za zemli mezhdu rodami Dzhalair i Turk, 1888.

TsGARUz, f. I-21, op. 1, d. 59: Ob iznasilovanii tuzemki Tuganai Suiarkulovoi, 1888.

TsGARUz, f. I-21, op. 1, d. 75: O nezakonnykh deistviiakh Narodnogo sud'i no. 2 aula Ata-Kurganskoi volosti Aktana Utenova, 1889.

TsGARUz, f. I-21, op. 1, d. 108: Po zhalobam zhitelei Iamskoivolostio zloupotrebleniiakh sud'i Mulla Khudai Nazara Sarybaeva toi zhe volosti, 1890-2.

TsGARUz, f. I-21, op. 1, d. 113: Po zhalobam zhitelei Narkustskoi volosti o vziatochnichestve narodnogo sud'i Bogdanskoi volosti, 1890.

TsGARUz, f. I-21, op. 1, d. 114: O vziatochnichestve narodnogo sud'i Magometa Turkebaeva Fistalitausskoi volosti, 1891-93.

TsGARUz, f. I-21, op. 1, d. 144: Postanovlenie Samarkandskogo oblastnogo pravleniia po obvineniiu narodonogo sud'i v nepravil'nom sostavlenii resheniia s"ezda narodnykh sudei, 1891.

TsGARUz, f. I-21, op. 1, d. 202: O vziatochnichestve narodnogo sud'i no. 1 aula Kizil-Kumskoi volosti Turganbeva, 1892.

TsGARUz, f. I-21, op. 1, d. 475: [Materialy o Tuia-Tartarskoi volosti].

TsGARUz, f. I-21, op. 1, d. 634. Po razboru spora o zemle mezhdu zhiteliami kishlaka Balgali, Iamskoi volosti, i zhiteliami Dzhalairskogo obshchestva toi zhe volosti, 1904.

TsGARUz, f. I-21, op. 1, d. 752: Po zhalobe kirgizki ob ubiistve ee rebenka, 1911.

TsGARUz, f. I-36 [Upravlenie Nachal'nika goroda Tashkenta], op. 1, d. 434: $O$ reshenii del mezhdu kirgizami podlezhashchikh sudu biev. 
TsGARUz, f. I-36, op. 1, d. 452: Po prosheniiu kaziev g. Tashkenta ob ostavlenii pri nikh Agliamov i Muftiev na prezhnem osnovanii, 1868.

TsGARUz, f. I- 36, op. 1, d. 454: untitled.

TsGARUz, f. I-36, op. 1, d. 725: O smerti Sibzarskogo kaziia i o nazhachenii na ego mesto drugogo litsa, 1870 .

TsGARUz, f. I-36, op. 1, d. 883: Proshenie zhitelei i svidetel'stva ob izbranii mestnykh dolzhnostnykh lits, 1873-4.

TsGARUz, f. I-36, op. 1, d. 2049: Opis' vakufov, medrese, mechetei, mazarov i o dokhodakh s nikh, n.d.

TsGARUz, f. I-36, op. 1, d. 2170: Kniga na zapisku reshenii kaziia Bish-agachskoi chastigor. Tashkenta s 29 Aprelia 1882 goda.

TsGARUz, f. I-36, op. 1, d. 2273: Po povodu predstavleniia rivaiata Voennomu Gubernatoru i Tainomu Sovetniku Girsu ob izmenenii poriadka torgovogo sbora i o naznachenii vzamen ego zakiatnogo sbora, 1883.

TsGARUz, f. I-36, op. 1, d. 2396: Spiski mull, medrese, prikaz po gorodu, spiski, prosheniia i perepiska o vybore i utverzhdenii muftiev, agliamov, mutavalliev $v$ g. Tashkente, 1884.

TsGARUz, f. I-36, op. 1, d. 2976: Perepiska s Syr-Dar'inskim oblastnym pravleniem o dostavlenii dokumentov vsekh vakufov g. Tashkenta v Syr-Dar'inskoe oblastnoe pravlenie, 1888.

TsGARUz, f. I-36, op. 1, d. 3367: O prichislenii Sibzarskogo narodnogo sud'i Mukhitdina Khodzhi $k$ otvetstvennosti za upushcheniia po vedeniiu opekunskikh del, 1887-93.

TsGARUz, f. I-36, op. 1, d. 3494: Ukaz Pravitel'stvennogo Senata i perepiska o raz'iasnenii prav gorodskikh sudei na sovershenii dokumentov gorozhan na zemliu, nakhodiashchuiusia $v$ Tashkentskom uezde, 1893.

TsGARUz, f. I-36, op. 1, d. 3587: Spisok vakufov, raporta, perepiska i vneseniia $v$ komitet blagotvoritel'nogo obshchestva s zemel'vakufa Nazarbiia i o drugikh vakufakh, 1895 .

TsGARUz, f. I-36, op. 1, d. 3708: Skhema i opis'vakufnykh zemel' Kukel'dash.

TsGARUz, f. I-36, op. 1, d. 3881: Reestry razbiraemykh del narodnymi sud'iami, proshenie o nedovol'stve resheniiami narodnykh sudei, 1898.

TsGARUz, f. I-36, op. 1, d. 4364: O vakufakh dvukh mechetei, sostoiashchikh v makhaliakh Makhsi-Duz Sibzarskoi chasti, 1907.

TsGARUz, f. I-36, op. 1, d. 6o83: untitled.

TsGARUz, f. I-36, op. 1, d. 6oog: Proekt uprazdneniia narodnykh sudov $v$ Turkestanskom krae, 1914.

TsGARUz, I-36, op. 1, d. 6487: S perepiskoi po vakufnym delam o naznachenii mutavalliev, ob iz'iatii uchastkov zemli, 1914. 
TsGARUz, I-36, op. 1, d. 6864: S perepiskoi, kasaiushcheisia vakufov, prosheniia na nepravil'nye resheniia sudei ob iz'iatii vladenii, o naznachenii novykh mutavalliev i t.p. perepiska, 1915.

TsGARUz, f. I-125 [Kantseliaria Khana Khivinskogo], op. 1, d. 29: O narushenii nekotorogo punkta torgovogo $i$ mirnogo dogovora Rossii $s$ Khivoi khivinskikh sanovnikami, ob ugone i krazhakh skota, o plennykh turkmen, $1883-85$.

TsGARUz, f. I-125, op. 1, d. 81: Perepiska ob arenduemykh uchastkah zemli khivinsko i russko poddannymi, 1900-2.

TsGARUz, f. I-125, op. 1, d. 190: Perepiska o rassledovanii brachnykh del (neuplata kalyma, uvoz chuzhikh zhen i t.p.) mezhdu khivinsko i russko poddannymi, 1904-5.

TsGARUz, f. I-125, op. 1, d. 486: Kaziiskie dokumenty, 1811-1919.

TsGARUz, f. I-125, op. 1, d. 495: Obraztsy "fatva" shariatskikh reshenii, /rivoiaty/.

TsGARUz, f. I-125, op. 1, d. 498: Zaiavlenie naseleniia na imia iasaul-bashi, 1909-18.

TsGARUz, f. I-125, op. 1, d. 579: Instruktsiia kazy i raisu m. Astana Khiv. Khanst. poslannaia vizirem, 1910.

TsGARUz, f. I-125, op. 2, d. 14: Iarlik khivinskogo khana Seiid-MukhammadBakhadur-khana o naznachenii Shakh Murada, syna Sarykulia, na dolzhnost' biia $v$ kazakhskom plemeni, 1279/1862.

TsGARUz, f. I-125, op. 2, d. 633: Vyzov esaul boshi v rezidentsiiu khana khivinskogo otvetchikov po denezhnomu, dvizhimomu i nedvizhimomu imushchestvu, 1328/1910.

TsGARUz, f. I-126 [Upravlenie Kushbegi èmira bukharskogo], op. 1, d. 6: Iarliki bukharskogo emira Abd al-Akhada o naznachenii na dolzhnosti kazi, raisov, khakimovv vilaiety Bukharskogo khanstva, 1884-1910.

TsGARUz, I-126, op. 1, d. 11: Khodataistva mirshabov pered emirom o naznachenii dzharib-bashiv vilaiety Bukharskogo khanstva, n.d.

TsGARUz, f. I-126, op. 1, d. 20: Khodataistva bukharskikh chinovnikov pered emirom o naznachenii aksakalov $i$ aminov $v$ vilaiety $i$ tumany Bukharskogo khantsva, n.d.

TsGARUz, I-126, op. 1, d. 22: Khodataistva bukharskikh chinovnikov pered emirom o naznachenii aksakalov i aminov v vilaiety i tumany Bukharskogo khantsva, n.d.

TsGARUz, f. I-126, op. 1, d. 667: Ariza kazi koshbegi o postuplenii deneg za arendu vakufnykh zemel'v Bukharskom khanstve, n.d.

TsGARUz, f. I-126, op. 1, d. 689: Otchet kazi o postuplenii deneg za sdachu $v$ arendu vakufnoi zemli dakhiaki-amma. Raspiski kazi o poluchenii deneg $s$ arendatorov vakufnykh zemel, n.d. 
TsGARUz, f. I-126, op. 1, d. 746: Ariza kazi i raisov èmiru o deiatel'nosti amlakdarov po sboru pozemel'noi podati khissat al-kharadzh $v$ khanstve, n.d.

TsGARUz, f. I-126, op. 1, d. 754: Ariza bukharskikh chinovnikov koshbegi o vypolnenii ego ukazaniia po zapresheniiu vzyskaniia khizmatana i farsakh-puli vyshe ustanovlennoi normy, 1827-1909.

TsGARUz, f. I-126, op. 1, d. 759: Ariza bukharskikh chinovnikov èmiru o zhalobakh na amlakdarov i drugikh chinovnikov na nezakonnye deistviia, n.d.

TsGARUz, f. I-126, op. 1, d. 940: Mubarakname émirov Nasrully, Muzaffara i Abd al-Akhada o razbore konfliktov po vakfu, 1812-1904.

TsGARUz, f. I-126, op. 1, d. 967: Ariza bukharskikh chinovnikov koshbegi o vzyskanii s bukharskikh poddannykh gosudarstvennykh dolgov, 1883-1916.

TsGARUz, f. I-126, op. 1, d. 1003: Ariza bukharskikh chinovnikov koshbegi po zemel'nym voprosam, n.d.

TsGARUz, f. I-126, op. 1, d. 1729: Makhzar i rivaiat, zaverennye v kantseliarii kazi, n.d.

TsGARUz, f. I-126, op. 1, d. 1761: Ariza kazi émiru o razbore del ob oskorblenii $i$ izbienii $v$ prisutstvii pribyvshego iz rikaba makhrama i vyplate emu khizmatane, n.d.

TsGARUz, f. I-126, op. 1, d. 1762: Ariza kazi koshbegi o razbore del ob izbienii $i$ oskorblenii $v$ prisutstvii pribyvshego iz rikaba makhrama i vyplate emu khizmatane, 1879-1895.

TsGARUz, f. I-126, op. 1, d. 1765: Ariza kazi koshbegi o razbore del ob izbienii $i$ oskorblenii $v$ prisutstvii pribyvshego iz rikaba makhrama i vyplate emu khizmatane, n.d.

TsGARUz, f. I-126, op. 1, d. 1796: Ariza kazi èmiru ob ustanovlenii blagonadezhnosti lits, vystupaiushchikh na sudebnykh zasedaniiakh $v$ kachestve svidetelei, n.d.

TsGARUz, f. I-133 [Prokuror Tashkentskoi sudebnoipalaty, 1899-1918], op. 1, d. 175: O Dzhandzhal'skom narodnom sud'e Mulla Abduvali Abdulkasambaev po 216 st. ulozheniia o nakazanii.

TsGARUz, f. I-133, op. 1, d. 227: Po delu ob obv. Kokan-Kishlakskogo narodnogo sud'i Tailalbaeva i ego mirza Dzhalial-Bai-Ogli v prestuplenii predusmotrennom 362 st. ul. o nakazanii.

TsGARUz, f. I-133, op. 1, d. 1020: Perepiska po protestam na prigovory i razresheniia narodnykh sudov, 1910.

TsGARUz, f. I-133, op. 1, d. 1325: Po nabliudeniiu za delom o narodnom sud'e Butitane Narumbetove, 1913 .

TsGARUz, f. I-133, op. 1, d. 1546: Po obv. narodnogo sud'i osedlogo naseleniia ToiTiubinsko i Osman-Atinskoi volosti, Tash. Uezda Mulla-Ali Magomed Khudai Bergenova po 347, 348 i 362 st. ul. o nakazanii, 1914. 
TsGARUz, f. I-133, op. 1, d. 1570: O protestakh na prigovory i resheniia narodnykh sudov, 1914.

TsGARUz, f. I-134 [Prokuror Syr-Dar'inskogo oblastnogo suda], op. 1, d. 57: Protesta na resheniia narodnykh sudei, 1891.

TsGARUz, f. I-134, op. 1, d. 76: Protesta na resheniia narodnykh sudei, 1892.

TsGARUz, f. I-134, op. 1, d. 91: Protesta na resheniia narodnykh sudei, 1893.

TsGARUz, f. I-134, op. 1, d. 110: Protesta na resheniia narodnykh sudei, 1894.

TsGARUz, f. I-134, op. 1, d. 123: Protesta na resheniia narodnykh sudei, 1895.

TsGARUz, f. I-134, op. 1, d. 130: Protesta na resheniia narodnykh sudei, 1895.

TsGARUz, f. I-134, op. 1, d. 152: Protesta na resheniia narodnykh sudei, 1897.

TsGARUz, f. I-134, op. 1, d. 162: Protesta na resheniia narodnykh sudei, 1898.

TsGARUz, f. I-134, op. 1, d. 180: Protesta na resheniia narodnykh sudei, 1899.

TsGARUz, f. I-164 [Tashkentskii Kazi-Kalian], op. 1, d. 1: Iarliki kokandskogo khana o naznachenii $v$ Tashkente kazi-kalana, alama $i d r$. dukhovnikh lits, 1822-61.

TsGARUz, f. I-164, op. 1, d. 3: Naznachenie generalom Cherniaevym 73 dukhovnykh lits na razlichnye dukhovnye dolzhnosti po khodataistvu kazi-kaliana g. Tashkent, 1865 .

TsGARUz, f. I-164, op. 1, d. 5: Razlichnye gramoty, svidetel'stva Tashkentskogo kazi-kaliana, poluchennye ot tsarskogo pravitel'stva, 1865-91.

TsGARUz, f. I-164, op. 1, d. 6: Lichnye dokumenty kazi Mukhitdina o kupliprodazhe i prava sobstvennosti, 1882-92.

TsGARUz, f. I-164, op. 1, d. 7: Lichnye dokumenty kazi Mukhitdina (prekrashchenie iskovykh del-ibro), 1873-1911.

TsGARUz, f. I-164, op. 1, d. 13: Makhzar i rivoiiat (nachalo sudebnogo deloproizvodstva i stat'i shariata), 1822-92.

TsGARUz, f. I-164, op. 1, d. 23: Resheniia Tashkentskogo kaziia, 1864-1912.

TsGARUz, f. I-164, op. 1, d. 39: Zaiavlenie Tura-Khana Tura voennomu generalgubernatoru o naznachenii ego mutavalliem po prinadlezhashchemu pravu po rodu Khodzha-Akhrar, 1888.

TsGARUz, f. I-164, op. 1, d. 50: Dokumenty o vybore èllikbashi, 1871-19o6.

TsGARUz, f. I-164, op. 2, d. 2a: Biografiia Tashkentskogo uchenogo Seid Mukhammed Khakim Khodzha (Otets Kaziia Mukhitdina), n.d.

TsGARUz, f. I-318 [Tovarish prokuror Tashkentskogo okruzhnogo suda], op. 1, d. 44: Perepiska po zhalobam na reshenie nar. sudov, 1912.

TsGARUz, f. I-318, op. 1, d. 45: Po prosheniiam ob oprotestovanii reshenii narodnykh sudei, 1913 .

TsGARUz, f. I-318, op. 1, d. 46: Po prosheniiam ob oprotestovanii reshenii narodnykh sudei, 1915 .

TsGARUz, f. I-318, op. 1, d. 67: Po prosheniiam ob oprotestovanii reshenii narodnykh sudei, 1916. 
TsGARUz, f. I-318, op. 1, d. 68: O perepiske po prosheniiam ob otmene reshenii narodnykh sudei, 1914 .

TsGARUz, f. I-318, op. 1, d. 72: O perepiske po prosheniiam ob otmene reshenii narodnykh sudei, 1915.

TsGARUz, f. I-318, op. 1, d. 76: Po prosheniiam ob oprotestovanii reshenii narodnykh sudei, 1913.

TsGARUz, f. I-318, op. 1, d. 78: Nariad po prosheniiam ob oprotestovanii reshenii narodnykh sudei, 1914.

TsGARUz, f. I-318, op. 1, d. 84: Po obvineniiu narodnykh sudei Saibetova $i$ Balkhatiiarova, 1899 .

TsGARUz, f. I-323 [Sobranie vakufnykh dokumentov], op. 1, d. 26: ‘Askar B̄̄ Ināq madrasaining waqfìasī, Bukhara 1818.

TsGARUz, f. I-323, op. 1, d. 34: Waqfìa-yi madrasa-yi Sayyid Amīn Bāy, Bukhara 1864 .

TsGARUz, f. I-323, op. 1, d. 667: Waqfìa-yi Nazākat Pāchā, Bukhara 1915.

TsGARUz, f. I-323, op. 2, d. 81: Saidumukhamat Alikan svoim vysochaishchim ukazom naznachaet [text missing] Kazy-Kalanom g. Tashkenta, 1238/1822-23.

TsGARUz, f. I-323, op. 2, d. 87: Abdul Muzaffar Said Mukhamod Khudaerkhon Bakhodur svoim vysochaishchim ukazom o naznachenii kazy kalanom Tashkentskoi oblasti Said Makmuda Khodzha, 1263/1847.

TsGARUz, f. I-323, op. 2, d. 88: Said Amir Mukhamad Khudoer Bakhodir-khon svoim vysochaishim ukazom naznachaet èshona Makhmud Khodzha nezavisimim Kazy Kalanom Tashkentskoi oblasti, 1270/1853-54.

TsGARUz, f. I-323, op. 2, d. 89: Said Mukhammad Malla Khan vysochaishim ukazom naznachaet Makhmud Khodzhu ishana Kazi-Kalanom g. Tashkenta, 1275/1858-59.

TsGARUz, f. I-336 [Voennyi Gubernator $i$ komanduiushchii voiskami Turkestanskoi oblasti], op. 1, d. 14: Po raznym zhalobam Kirgiz i razbor po ètomu predmetu, n.d.

TsGARUz, f. I-365 [Kazii Sibzarskoi chasti gor. Tashkenta], op. 1, d. 74: Kniga na zapisku reshenii kaziia Sibzarskoi chasti gor. Tashkenta 1899 goda.

TsGARUz, f. I-365, op. 1, d. 85: Kniga reshenii kaziia Sibzarskoi chasti gor. Tashkenta, 1910.

TsGARUz, f. I-365, op. 1, d. 94: Narodnyi sud'ia Sibzarskoi chasti g. Tashkenta. Dukhovnoe zaveshchanie Seid Gazykhana Fatkhullakhana, 1913-14.

TsGARUz, f. I-717 [Sovet Turkestanskogo General-Gubernatora], op. 1, d. 6: Zhurnaly Soveta Turkestanskogo General-Gubernatora, 1891.

TsGARUZ, f. R-2773 [Lichnyi fond M.Z. Massona], op. 1, d. 1103: V.L. Viatkin, K voprosam izucheniia Uzbekov v Srednei Azii XVI vek [sic]. Unpublished manuscript, 1932. 
TsGARUz, f. R-2678 [Lichnyi fond O.D. Chekhovich], op. 1, d. 12: Iuridischeskie zakliucheniia, n.d.

TsGARUz, f. R-2678, op. 1, d. 6o: Istoriia razvitiia aktov iuridicheskogo oformleniia feodal'nykh otnoshenii v Srednei Azii XII-XVI vv. Unpublished manuscript, 1979

TsGARUz, R-2678, op. 1, d. 167: "Risola-i Khabibiia." (Traktat o zemliakh desiatichnykh i kheradzhnykh). Perevod sochineniia Ibadellakha ibn Khodzhaarifa al-Bukhary s persidskogo iazyka (rukopisi no. 4976 IV Uz). Chernovoi avtograf. Prilozhenie: Faksimile rukopisi no. 4976, 1963.

TsGARUz, R-2678, op. 1, d. 379: Vypiski i perevody iz formuliarnika "Mukhtar al'Ikhtiiara" Ikhtiiara ibn Giiasuddina al Khusaini iz rukopisi no. A-933, n.d.

TsGARUz, R-2678, op. 1, d. 381: Vypiska iz raporta Kuna A. s perevodom spiska mulkov Zeravshanskogo okruga ot 20 fevralia 1870 g. Rukopis.' Prilozhenie: Spisok mulkov, sostavlennyi chinovnikom Bukharskogo emira Muzaffara, n.d.

TsGARUz, f. R-2678, op. 1, d. 531: Pis'ma Davidovich E.A. Chekhovich O.D., 1956-81.

TsGARUz, f. R-2678, op. 2, d. 4: Iuridicheskoe zakliuchenie (rivoiat) musul'manskikh zakonovedov (muftiev) o rezul'tatakh vizual'nogo osmotra tela umershego mal'chika. 1800 (na pechati).

TsGARUz, f. R-2678, op. 2, d. 17: Iuridicheskoe zakliuchenie o prioritete cheloveka, obrabatyvaiuschego zemliu $v$ prave rasporiazhatsia eiu. 1904 (na pechati).

TsGARUz, f. R-2678, op. 2, d. 9o: Kupchaia Astanakula Kushbegi, n.d.

TsGARUz, f. R-2678, op. 2, d. 126: Iuridicheskoe zakliuchenie o nevozmozhnosti otkaza ot svoikh prezhnykh pokazanii, sdelannykh v prisutstvii kaziia (sud'i), 1864 g. (na pechati).

TsGARUz, f. R-2678, op. 2, d. 177: Kollektsiia aktov Andreeva M.S., n.d.

TsGARUz, f. R-2678, op. 2, d. 178: Kollektsiia aktov Andreeva M.S., n.d.

TsGARUz, R- 2678, op. 2, d. 244: Formuliarnik iuridicheskikh [sic] dokumentatsii $X X$ v. na tadzhikskom iazyke, arabskim shriftom [1910 g.]

TsGARUz, R-2678, op. 2, d. 251: Qārī Aḥmad, Tarjuma-yi aḥwāl-i QāḍīKalānhāa-yi darūn-i Bukhārā, 1940.

\section{Khiva}

IQM, no. 2053: Iarlik Allakuli khana o naznachenii kaziem goroda Vazira Mully Kurully, 1833 .

IQM, P-8, kp 3674, ll. 33-33ob, n.d. 


\section{Khujand}

ObAKh, f. I-145, op. 1, d. 58, ll. 1-20: Kollektsiiai fondi shaxsii Mullomuhammad Sharif ibni Abduzalil [Mullā Muḥammad 'Aẓīm Mullā Muḥammad Sharīfūghlī]-qozii volosti Mahram.

\section{Nukus}

FBKOANRUz, no. R-9o: S.K. Kamalov, Khoziaistvo karakalpakov XIX v, notes and documents, n.d.

\section{Samarqand}

AMIKINUz, no. 828: M.S. Iusupov, Sud v Bukhare. Sudoustroistvo i sudoproizvodstvo $v$ Bukharskom emirate $v$ kontse XIX i nachale XX v.v. Unpublished manuscript, 1941.

AMIKINUz: Untitled collection of Arabic-script documents. 